\section{Wheat germ agglutinin staining as a suitable method for detection and quantification of fibrosis in cardiac tissue after myocardial infarction}

\section{B. Emde, A. Heinen, A. Gödecke, K. Bottermann}

Department of Cardiovascular Physiology, Heinrich-Heine-University Düsseldorf, Germany

\begin{abstract}
The quantification of fibrotic tissue is an important task in the analysis of cardiac remodeling. The use of established fibrosis staining techniques is limited on frozen cardiac tissue sections due to a reduced color contrast compared to paraffin embedded sections. We therefore used FITC-labeled wheat germ agglutinin (WGA), which marks fibrotic tissue in comparable quality as the established picrosirius red (SR) staining, for the staining of post myocardial infarction scar tissue. The fibrosis amount was quantified in a histogrambased approach using the non-commercial image processing program ImageJ. Our results clearly demonstrate that WGA-FITC is a suitable marker for cardiac fibrosis in frozen tissue sections. In combination with the histogram-based analysis, this new quantification approach is i) easy and fast to perform; ii) suitable for raw frozen tissue sections; and iii) allows the use of additional antibodies in coimmunostaining.
\end{abstract}

\section{Introduction}

Cardiac fibrosis is a typical phenomenon during cardiac remodeling due to several cardiac pathologies including post myocardial infarction (MI). To investigate the pathomechanisms of this highly prevalent disease many experimental mouse models as permanent or temporary coronary artery occlusion are commonly used in the field of cardiovascular research. ${ }^{1,2}$ In these models, the post MI process of cardiac remodeling is accompanied by the formation of fibrotic tissue, which plays a critical role in the development of the postMI systolic and diastolic cardiac function. Therefore, the detection and quantification of fibrotic tissue in the myocardium are crucial for the analysis of post MI cardiac remodeling.

Several histological techniques have been established for the staining of fibrosis and scar formation in cardiac tissue sections. A standard method for the detection of fibrotic tissue and scar formation within the myocardium is the picrosirius red staining. ${ }^{3}$ In 2008 Gaspard and Pasumarthi ${ }^{4}$ published a method for the quantification of diffuse cardiac fibrosis in picrosirius red/fast green stained cardiac tissue. However, for automated quantification this method requires a high color contrast, which is reduced if picrosirius red staining is applied to cryosections in comparison to paraffin embedded tissue. In addition, the benefit of picrosirius red staining is further limited by the fact that co-immunostaining with antibodies, which allow co-localization studies or the analysis of several cell compartments in one approach, are not possible. Therefore, a simple method for the staining and quantification of post MI fibrosis in frozen heart tissue sections, which is also compatible with co-immunostaining, is of high interest. Wheat germ agglutinin (WGA), which binds to glycoproteins of the cell membrane, is routinely used for the staining of skeletal and cardiac sarcolemma to determine cross sectional area or myocyte density. In this context we observed that WGA also labeled fibrotic tissue in cardiac sections after myocardial infarction. Thus, we systematically analyzed in this study WGAs potential for the staining and quantification of post MI scar formation.

\section{Materials and Methods}

\section{Experimental myocardial infarction}

All animal experiments were performed in accordance with the national guidelines on animal care and approved by the Bezirksregierung Düsseldorf.

Twelve-week-old C57Bl6 male mice (Janvier, Saint-Berthevin Cedex, France) were used. After induction of anesthesia (isoflurane $2.5 \%$ ), mice were endotracheally intubated and ventilated using a small rodent ventilator (MiniVent Type 845, Hugo Sachs Elektronik, March-Hugstetten, Germany). Anesthesia was maintained by continuous administration of $2 \%$ isoflurane. Additionally, buprenorphine $(0.1 \mathrm{mg} / \mathrm{kg})$ was injected subcutaneously before the start of the surgical procedure. Afterwards, a left thoracotomy at the fourth intercostal space was performed, and the left anterior descending coronary artery (LAD) was exposed. After identification of the $\mathrm{LAD}$, a 7-0 prolene suture was passed around the LAD approximately one millimeter distal of the left atrium. Myocardial ischemia was induced by occlusion of the $\mathrm{LAD}$ and verified by ECG (electrocardiogram) ST-segment elevation and paleness of the myocardial area at risk (AAR). After 45 min the LAD was reopened and ade-
Correspondence: Dr. Katharina Bottermann, Department of Cardiovascular Physiology, Heinrich-Heine-University Düsseldorf, Universitätstr. 1, 40225 Düsseldorf, Germany.

Tel. +49.211 .8112445 - FAX: +49.211 .8112672 .

E-mail: katharina.bottermann@uni-duesseldorf.de

Key words: Cardiac fibrosis, myocardial infarction, wheat germ agglutinin, fibrosis quantification, histogram-based analysis.

Contributions: $\mathrm{BE}, \mathrm{KB}$, histological preparations and staining; $\mathrm{AH}$, I/R-experiments; $\mathrm{BE}, \mathrm{AH}, \mathrm{AG}$ $\mathrm{KB}$, study design, data analysis, writing and revision of the manuscript.

Conflicts of interest: the authors declare no conflicts of interest.

Acknowledgments: we thank Dr. Annika Raupach, Lucia Leitner and Leonie Windeln (Department of Cardiovascular Physiology, Heinrich-HeineUniversity Düsseldorf, Germany) for planimetric analysis and Nina Hölscher (Department of Anesthesiology, Heinrich-Heine-University Düsseldorf, Germany) for the paraffin embedded tissue sections, shown in Figure 1.

Received for publication: 29 August 2014. Accepted for publication: 1 December 2014.

This work is licensed under a Creative Commons Attribution NonCommercial 3.0 License (CC BYNC 3.0).

(C) Copyright B. Emde et al., 2014

Licensee PAGEPress, Italy

European Journal of Histochemistry 2014; 58:2448 doi:10.4081/ejh.2014.2448

quate reperfusion was verified by reversion of ECG changes and occurrence of epicardial hyperemia. Subsequently, the suture was removed and the chest was closed. After cessation of isoflurane administration, mice were extubated after restart of spontaneous breathing. Four weeks after myocardial infarction, animals were sacrificed and hearts were removed for histological analysis.

\section{Histology}

After myocardial infarction and four weeks of reperfusion, hearts were either paraffin embedded or the raw material was snap frozen at $-40^{\circ} \mathrm{C}$ in isopentane. In paraffin sections (4 $\mu \mathrm{m})$ the wax was dissolved by an organic solvent and the tissue slices rehydrated before picrosirius red stain was applied. Cryosections $(8 \mu \mathrm{m})$ from raw snap frozen tissue were fixed in $4 \%$ paraformaldehyde (PFA) in $0.1 \mathrm{M}$ sodium phosphate buffer (PB) pH 7.4 or Zambonis fixative $(0.1 \mathrm{M} \mathrm{PB}, 4 \%$ (w/v) PFA, $15 \%$ (v/v) picric acid) for $10 \mathrm{~min}$. Washing steps were performed in PBS, PBS/0.1\% Saponin or in 
PBS/0.2\% Tween 20 according to the further requirements. Picrosirius red (SR) staining was performed according to the protocols of Junqueira et al. ${ }^{3}$ and Sweat et al. ${ }^{5}$

Wheat germ agglutinin (WGA) labeling was routinely used in combination with the secondary antibody in immunohistochemical preparations. Lectin from triticum vulgaris FITC conjugate (\# L4895, Sigma-Aldrich, St. Louis, M0, USA) was diluted 1:100 $(10 \mu \mathrm{g} / \mathrm{mL})$ in the required buffer. Incubation time was one hour protected from light. After three washing steps, the sections were coverslipped with a water-soluble antifading mounting medium. Collagen I staining was performed using an anti-collagen I antibody (ab34710, Abcam, Cambridge, UK) diluted 1:100 in the required buffer. As secondary antibody antiRabbit-Cy3 (111-165-144, Jackson Dianova, Hamburg, Germany) was used in a concentration of $2.5 \mu \mathrm{g} / \mathrm{mL}$.

Slides were analyzed with fluorescence microscope Keyence BZ 9000 (Keyence, NeuIsenburg, Germany). All shown images were taken with $4 \mathrm{x}$ objective using the merge function, as not otherwise specified.

\section{Quantification of scar size after MI by planimetry}

The analysis of scar formation was performed using the non-commercial image processing software ImageJ (http://imagej. nih.gov/ij/). For this, the measure function of Image J was used on images of both picrosirius red and WGA-FITC stained cryosections. The scar sizes and the areas of all five analyzed whole hearts were traced by five independent investigators in both SR and WGA-FITC stained hearts. The scar size is expressed as percent of the whole heart. Data are presented as mean \pm standard deviation (SD). To test for a possible correlation between either planimetric analysis of SR $v s$ WGA-stained hearts, or histogram vs planimetric assessed scar size of WGA stained hearts, linear regression analysis was performed using Microsoft Excel. $\mathrm{R}^{2}$ and $\mathrm{P}$ values are given in the respective figure legends.

\section{Results}

\section{Picrosirius red staining on paraffin embedded sections and raw frozen tissue}

We first compared picrosirius red stained post-MI heart sections, which were either paraffin embedded or from raw frozen tissue. Figure 1 shows, that the color contrast between the red stained collagen strings and the yellow stained background, was much
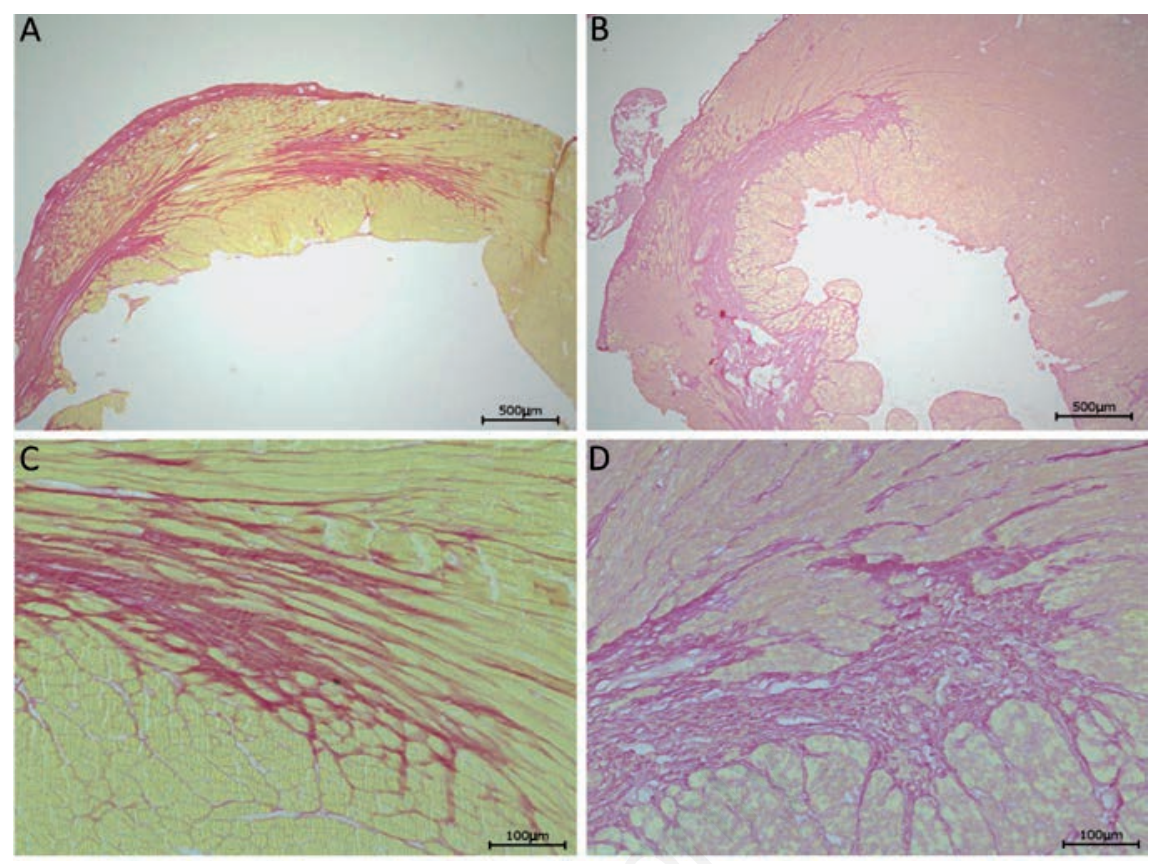

Figure 1. Comparison of picrosirius red staining of paraffin embedded (A,C) and raw frozen tissue $(B, D)$. Images were taken with $4 x$ objective $(A, B)$ and $20 x$ objective $(C, D)$ of post-MI heart sections where a part of the scar (dark red dye) and the remote myocardium (yellow dye) is visible.
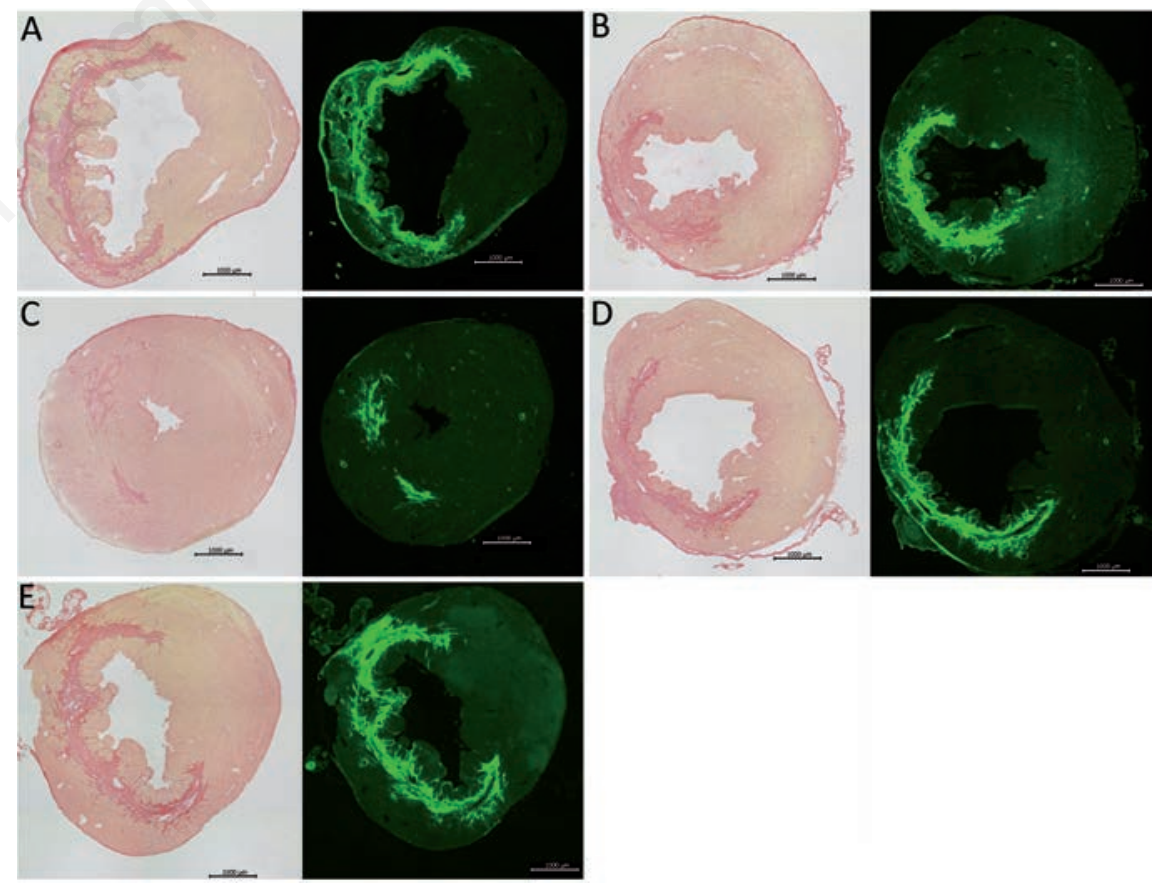

Figure 2. Sirius red stain (left image) and WGA-FITC stain (right image) of mouse heart frozen tissue sections, after $45 \mathrm{~min}$ ischemia and four weeks reperfusion. All hearts show a scar in the myocardium of the left ventricle in different dimensions. 
higher in paraffin embedded sections (Figure $1 \mathrm{~A}, \mathrm{C}$ ) as on raw frozen tissue (Figure $1 \mathrm{~B}, \mathrm{D}$ ).

\section{Quantification of scar size by planimetry}

As a high color contrast is an important requirement for automated quantification of fibrotic tissue, we next examined WGA-FITC staining on raw frozen tissue and compared its ability to mark post-MI scar formation with picrosirius red staining.

To determine the scar size after MI, cryosections of all hearts, which underwent $45 \mathrm{~min}$ regional myocardial ischemia followed by four weeks of reperfusion, were stained by both SR and WGA. The heart sections after SR (left image) and WGA-FITC staining (right image) are shown in Figure 2. The SR staining marks the fibrotic tissue within the scar with a dark red dye; the remote myocardium, i.e. the noninfarcted tissue, appears in a slight red to yellow color. Obviously, WGA-FITC staining marked comparable regions of the left ventricle in a bright green fluorescent color. To validate whether scar size labeling is congruent after both staining protocols, we analyzed scar size by planimetry of both, SR and WGA-FITC stained cryosections.

Our results show a strong correlation of $\mathrm{R}^{2}=0.96(\mathrm{P}=0.004)$ between the infarct sizes determined by planimetry from SR and WGAFITC stained frozen heart sections (Figure 3). These data clearly show that FITC-labeled WGA staining can be used for reliable quantification of scar formation after myocardial infarction. For further characterization of WGA-binding to extracellular matrix, additional co-staining of WGA with anti-collagen I-antibody was also performed exemplarily. Figure 4A shows the overlay of WGA and collagen I staining, which clearly demonstrates, that both, the lectin and the antibody stain components of the extracellular matrix, in these slides mainly the scar. Nevertheless, higher magnifications (Figure 4 $\mathrm{B}, \mathrm{C})$ reveal, that they do not produce a typical yellow merge image, suggesting different binding sites of WGA and anti-collagen antibody in the extracellular matrix.

\section{Quantification of fibrotic tissue by histogram analysis}

A drawback of scar size analysis by planimetry is the fact that fibrotic areas can be underor overestimated by tracing them. For instance, the section in Figure 2A shows much diffuse fibrotic tissue within the border zone of the ischemic area, which is however not covered by planimetric analysis, as this only covers the substantial scar. Therefore the planimetry rather underestimates the fibrosis amount of this section. On the other hand, in Figure 2C the substantial scar has areas, which do not show fibrosis and which leads

A
\begin{tabular}{|l|l|l|l|l|l|l|l|l|l|l|}
\hline & \multicolumn{2}{|c|}{ A } & \multicolumn{2}{|c|}{ B } & \multicolumn{2}{|c|}{ C } & \multicolumn{2}{|c|}{ D } & \multicolumn{2}{|c|}{ E } \\
\hline & Mean & SD & Mean & SD & Mean & SD & Mean & SD & Mean & SD \\
\hline WGA, P & 13.69 & 2.58 & 9.30 & 1.38 & 5.84 & 1.67 & 11.94 & 1.23 & 21.85 & 3.54 \\
\hline SR, P & 14.26 & 1.06 & 6.78 & 0.83 & 4.85 & 0.96 & 10.42 & 0.84 & 19.45 & 2.82 \\
\hline
\end{tabular}

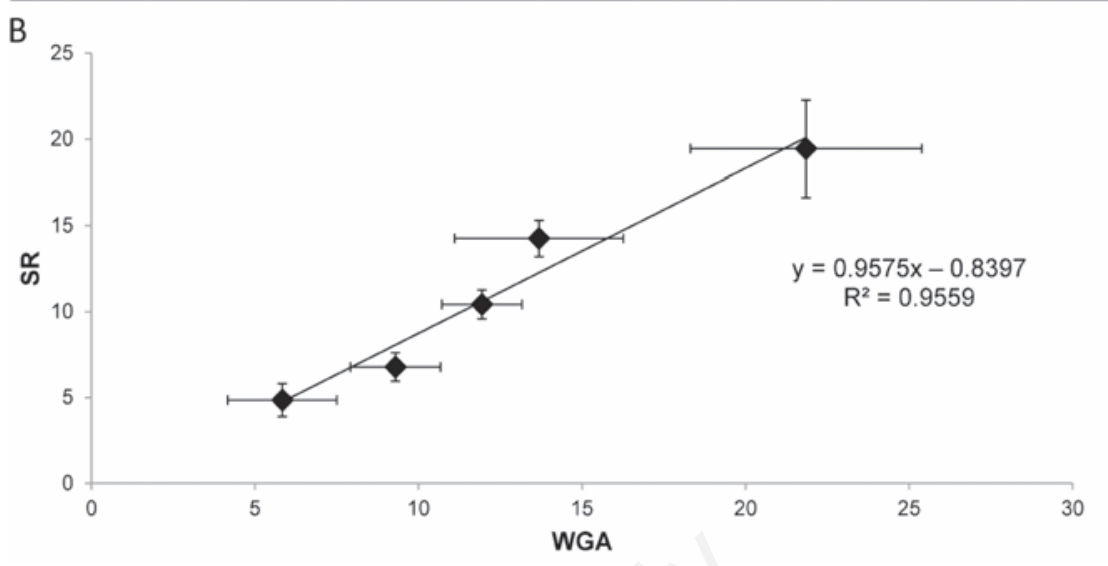

Figure 3. A) Comparison of numerical values of planimetric scar size analyses between wheat germ agglutinin-FITC (WGA, P) and picrosirius red (SR, P) stained heart cryosections. $\mathrm{B})$ Correlation diagram of these data. $\mathrm{R}^{2}=0.9559, \mathrm{P}=0.004$.
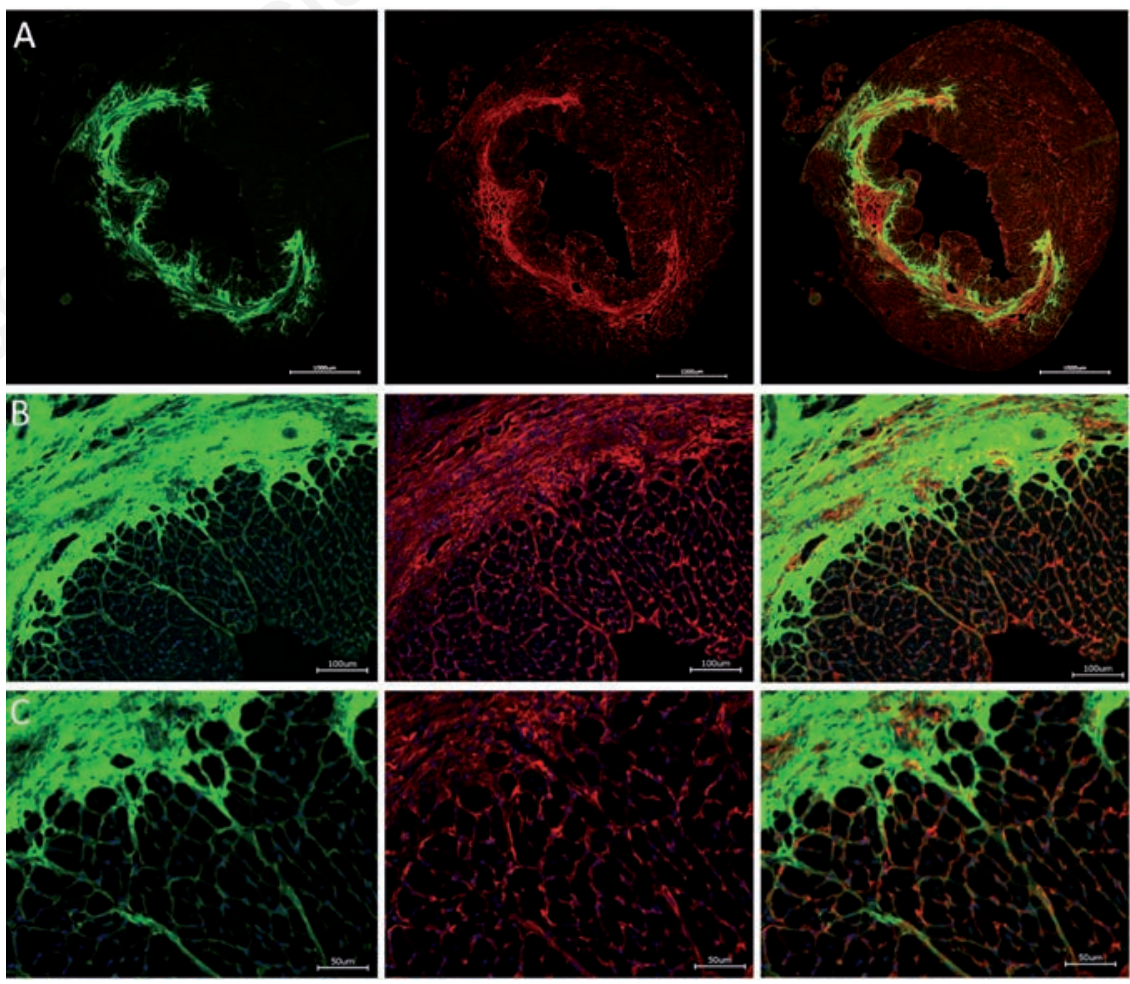

Figure 4. A) WGA (green) and Collagen I (red) co-staining of heart slices of one exemplary I/R-experiment. B) 20x magnification of WGA/Collagen I co-stained heart showing scar tissue and normal tissue. Nuclei are stained with DAPI (blue). C) 40x magnification of a WGA/Collagen I co-stained heart. Nuclei are stained with DAPI (blue). Staining in all panels from left to right: WGA (green, FITC), Collagen I (red, Cy3), Overlay. 
therefore rather to an overestimation of fibrosis amount. To avoid this, we developed a computer based quantification approach for fibrotic tissue within WGA-FITC stained post MI cryosections. We performed a histogram-based quantification approach using the histogram function of ImageJ on jpeg-images. Within a chosen area, these histograms show the distribution of the pixels to 255 brightness or intensity levels. The green channel was used for WGA staining and the red channel for SR staining, respectively. The background brightness level was determined using a $1 \mathrm{~mm}^{2}$ area within the septum of the heart, i.e. an area where no ischemia and therefore no fibrosis occurred (Figure 5A). A histogram of this background area was achieved and compared to a histogram obtained from the complete heart. Subsequently, the pixel distribution of the background area was extrapolated to the pixel amount of the whole heart. Figure $5 \mathrm{C}$ shows exemplarily for one I/R (ischemia/reperfusion)-experiment the two histograms of the whole heart (red line) and the extrapolated background (black line). To calculate the percentage of fibrosis the pixel amount of the whole heart ranging above the background (black arrow) was referred to the complete pixel amount of the heart. The histogram analysis of WGA-FITC stained heart A (Figure 5C) shows a large overlap between background histogram (black border) and complete heart histogram (red border) in the range between brightness level 25 and 70 . Pixels with higher light intensities (brightness level 70 to 255) were only detected in the complete heart histogram. Therefore, these pixels represent the fibrotic tissue. After background subtraction, a fibrosis amount of $23.6 \%$ was calculated for this heart.

We also attempted to apply this histogrambased quantification approach to SR stained sections however, this failed (Figure $5 \mathrm{~B}, \mathrm{D}$ ). The histograms of the SR staining in Figure 5D show a big overlap between the background and the whole heart histogram in the range between level 190 and 250. No greater distribution of the pixels in the whole heart can be detected, as after WGA-FITC stain. Therefore it was not possible to calculate a fibrosis amount from histograms derived from SR staining of these cryosections.

\section{Planimetric vs histogram-based analysis}

As mentioned above, histogram-based analysis of SR stained sections was not possible. To compare the sensitivity of planimetric and histogram-based analysis in quantification of fibrotic tissue, we therefore calculated the correlation between both methods in WGAFITC stained cryosections (Figure 6). A deter-
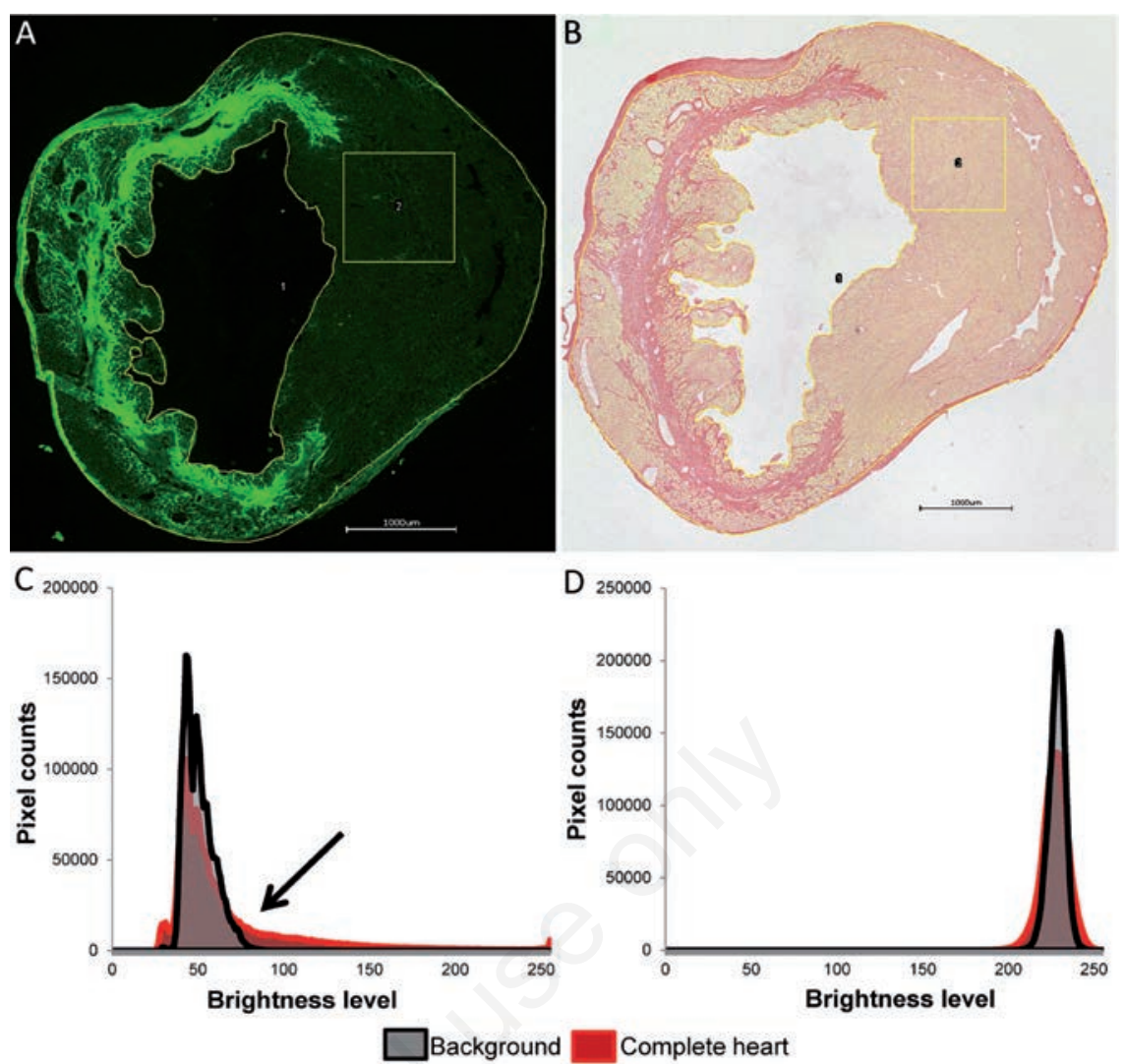

Figure 5. Histogram-based quantification of fibrosis amount. A,B) Exemplary traces of the background area and the complete heart for WGA-stained (A) and SR-stained (B) section. C) Overlay of the green histograms of the background (black border) and of the complete heart (red border). The Background was extrapolated to the pixel amount of the whole heart for a direct comparison. From brightness level -70 (black arrow) the pixel of the whole heart over the background represent the fibrotic fraction. D) Red histograms of the heart after SR staining for background (black border) and complete heart (red border) with extrapolated background as described above.

\section{A}

\begin{tabular}{|l|l|l|l|l|l|l|l|l|l|l|}
\hline \multicolumn{3}{|l|}{ A } & B & C & D & E & \\
\hline & Mean & SD & Mean & SD & Mean & SD & Mean & SD & Mean & SD \\
\hline WGA, P & 13.69 & 2.58 & 9.30 & 1.38 & 5.84 & 1.67 & 11.94 & 1.23 & 21.85 & 3.54 \\
\hline WGA, H & 23.55 & - & 15.44 & - & 3.39 & - & 19.25 & - & 28.10 & - \\
\hline
\end{tabular}

B

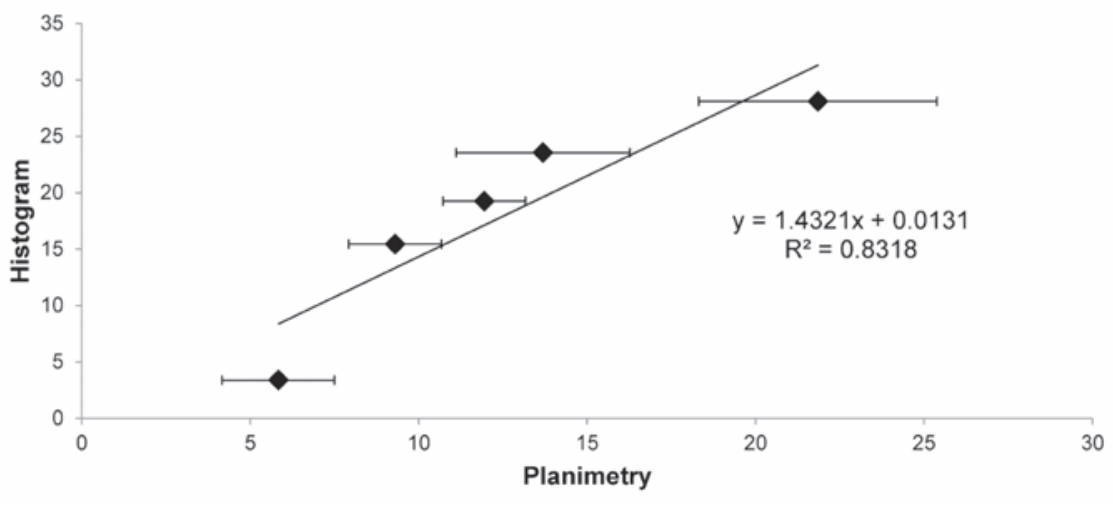

Figure 6. A) Comparison of numerical values of planimetric (WGA, P) and histogrambased (WGA, H) analysis of fibrosis amount in all five examined hearts after WGA-FITC staining. B) Correlation diagram of the planimetric and histogram-based automatically assessed data. $R^{2}=0.8318, P=0.0309$. 
mination coefficient of $\mathrm{R}^{2}=0.83 \quad(\mathrm{P}=0.0309)$ was achieved, mostly with higher values in the histogram-analyzed results. This points to a higher sensitivity of the computer-based method over the planimetric analysis.

\section{Discussion}

The easy and fast quantification of fibrotic tissue is an important task in the histological analysis of cardiac remodeling in animal models. In the past several methods have been established to address this issue. A typical fibrosis staining method is the picrosirius red stain. ${ }^{3}$ A couple of years ago, Gaspard and Pasumarthi ${ }^{4}$ published a color-subtractive computer assisted image analysis (CS-CAIA)based quantification method for diffuse fibrosis on sirius red/fast green stained tissue sections. However, this quantification approach requires a high color contrast, and is therefore poorly assignable to raw frozen tissue, which shows a reduced color contrast after picrosirius red staining compared to e.g. paraffin embedded sections, as shown in Figure 1.

In this study we present a new staining and histogram-based quantification approach for fibrotic tissue within the heart using FITClabeled wheat germ agglutinin, which i) can be used for reliable quantification of scar formation after myocardial infarction in mice; ii) allows co-immunostaining with additional antibodies; and iii) is suitable for frozen tissue sections. WGA is a lectin originally derived from wheat, which binds to $\mathrm{N}$-acetylglucosamin and sialic acid. ${ }^{6,7}$ The fact that lectins can bind to carbohydrates, which are present on the cell surface, is known for more than 30 years. In combination with a histological label as horse radish peroxidase or fluorescent dyes, lectins are very suitable markers for cell membranes. ${ }^{8-10}$ Next to the labeling of the glycocalix, it was also reported that WGA binds to connective tissue and extracellular matrix. ${ }^{11-13}$ Components of the extracellular matrix, for example glycosaminoglycans as hyaluronic acid, are rich in $\mathrm{N}$-acetylglucosamin, providing a rationale to use WGA for detection of scar tissue after myocardial infarction. The results of the present study clearly demonstrate that myocardial fibrotic tissue can be stained by WGA-FITC in cryosections. We found a correlation of $\mathrm{R}^{2}=0.96$ between planimetric determined post-MI scar size after both, SR and WGA-FITC staining. This indicates that WGAFITC is a suitable marker for fibrotic tissue. Furthermore, WGA-staining allows co-staining with e.g. anti-collagen I antibody for a structural differentiation of the extracellular matrix.
Figure 4 demonstrates that parts of the scar are stained by either collagen I antibody or WGA in different intensities indicating a regional heterogeneity of different matrix components within the scar. With WGA/collagen I co-staining a differentiation and categorization of the extracellular matrix components can be performed.

In addition, we tested the ability to quantify the cardiac fibrosis amount with histograms of WGA-FITC and SR stained heart sections. Our results show that this histogram-based approach is suitable for the WGA-FITC label while the application on picrosirius red staining failed. A possible explanation might be the reduced color contrast due to elevated red background color of picrosirius red staining on cryosections. Nevertheless, WGA-FITC stains, differently from picrosirius red, also the cell membranes (Figure $4 \mathrm{~B}, \mathrm{C}$ ), leading to a background fluorescence, which has to be subtracted for the quantification of the fibrosis amount. Interestingly, we also found the histogram-based quantification of fibrosis to be more sensitive than the planimetric approach. The poor correlation between these methods applied to WGA-FITC stained sections $\left(R^{2}=0.83\right)$ was due to higher fibrosis levels detected with the histogram-based approach. This higher sensitivity might be due to the fact that fibrosis also occurs afar from the substantial scar, i.e. in a diffuse form within the ischemic border zone. With the planimetry, it is not possible to cover this diffuse fibrosis and therefore this method rather underestimates the amount of fibrosis in post-MI hearts.

Taken together, there are several advantages for the use of the WGA-FITC stainingand quantification-approach as reported here, in comparison to other fibrosis staining and quantification methods. First, on raw frozen material common staining techniques like picrosirius red often do not show a high contrast between fibrotic and non-fibrotic tissue, as they do on paraffin-embedded tissue. However, this is a critical requirement for an automated quantification approach. On the contrary, WGA-FITC staining shows a high contrast and is therefore more suitable for this application. Second, the use of WGA-FITC allows the application of immunohistochemical staining with additional antibodies in the same approach, which is not possible in combination with histological preparations. Third, the use of WGA-FITC staining in combination with a histogram-based quantification is easy and fast to perform and therefore a competitive technique for the analysis of fibrosis amount on raw frozen tissue sections.

\section{References}

1. Klocke R, Tian W, Kuhlmann MT, Nikol S. Surgical animal models of heart failure related to coronary heart disease. Cardiovasc Res 2007;74:29-38.

2. Houser SR, Margulies KB, Murphy AM, Spinale FG, Francis GS, Prabhu SD, et al. Animal models of heart failure: a scientific statement from the American Heart Association. Circ Res 2012;111:131-50.

3. Junqueira LCU, Bignolas G, Brentani RR. Picrosirius staining plus polarization microscopy, a specific method for collagen detection in tissue sections. Histochem $\mathrm{J}$ 1979;11:447-55.

4. Gaspard GJ, Pasumarthi KB. Quantification of cardiac fibrosis by colour-subtractive computer-assisted image analysis. Clin Exp Pharmacol Physiol 2008;35:67986.

5. Sweat F, Puchtler H, Rosenthal, I S. Sirius red F3BA as a stain for connective tissue. Arch Pathol 1964;78:69-72.

6. Bhavanandan VP, Katlic AW. The interaction of wheat germ agglutinin with sialoglycoproteins. The role of sialic acid. J Biol Chem 1979;254(10):4000-8. http://www. jbc.org/content/254/10/4000

7. Monsigny M, Roche AC, Sene C, MagetDana R, Delmotte F. Sugar-lectin interactions: how does wheat-germ agglutinin bind sialoglycoconjugates? Eur J Biochem 1980;104:147-53.

8. Gonatas NK, Avrameas S. Detection of plasma membrane carbohydrates with lectin peroxidase conjugates. J Cell Biol 1973;59:436-43.

9. Huet C, Garrido J. Ultrastructural visualization of cell-coat components by means of wheat germ agglutinin. Exp Cell Res 1972; 75:523-7.

10. Pena SD, Gordon BB, Karpati G, Carpenter S. Lectin histochemistry of human skeletal muscle. J Histochem Cytochem 1981;29: 542-6.

11. Ohno J, Tajima Y, Utsumi N. Binding of wheat germ agglutinin in the matrix of rat tracheal cartilage. Histochem J 1986;18: 537-40.

12. Söderström KO. Lectin binding to collagen strands in histologic tissue sections. Histochemistry 1987;87:557-60.

13. Kostrominova TY. Application of WGA lectin staining for visualization of the connective tissue in skeletal muscle, bone, and ligament/tendon studies. Microsc Res Tech 2011;74:18-22. 